\title{
EDITORIAL
}

\section{Sudden unexpected death in people with down syndrome and epilepsy: another piece in this complicated puzzle}

\author{
Carla A. Scorza,' Fulvio A. Scorza,' Ricardo M. Arida," Esper A. Cavalheiro,,III \\ 'Disciplina de Neurologia Experimental, Universidade Federal de São Paulo-Escola Paulista de Medicina (UNIFESP/EPM). São Paulo, Brasil. "Departamento \\ de Fisiologia. Universidade Federal de São Paulo/Escola Paulista de Medicina (UNIFESP/EPM). São Paulo, Brasil. "'Associação de Pais e Amigos dos \\ Excepcionais de São Paulo (APAE-SP). São Paulo, Brasil.
}

A review of the literature shows that we owe the first medical description of a child with Down syndrome to JeanÉtienne Dominique Esquirol in 1838. It would be 28 years until John L. Down published a paper accurately describing the characteristics of this syndrome, which is therefore named after him. ${ }^{1}$ In 1959, Lejeune and Jacobs independently determined that Down syndrome was linked to trisomy 21. ${ }^{2}$ At the time of publication, Down syndrome is the most common chromosomal abnormality, causing intellectual disability with intelligence quotients (IQs) in the range of $20-85 .^{3}$ In general terms, there is no racial predilection, the male to female ratio is approximately $1.15: 1$, and it occurs in approximately 1 in 500 to 1 in 1000 live births. ${ }^{3,4}$ Furthermore, Down syndrome may present considerable challenges, as manifestations may be quite diverse and involve many organ systems, which may be related to the cases of sudden death occurring in this group. ${ }^{3}$ Moreover, we must point out that other diseases underlying Down syndrome may be directly associated with the fatal episodes observed in these individuals. Epilepsy occupies an interesting role in this scenario. To better understand this new paradigm, some hypotheses must be put forward.

Epilepsy is one of the most common serious brain disorders. Worldwide, epilepsy is estimated to affect 50 million people, most of whom live in developing countries and therefore may have limited access to medical treatment. ${ }^{5,6}$ Unfortunately, epilepsy is associated with a two- to three-fold increase in mortality compared with the general population, and sudden unexpected death is the most important direct epilepsy-related cause of death. ${ }^{7}$ In general terms, sudden unexpected death in epilepsy is responsible for $7.5 \%$ to $17 \%$ of all deaths in epilepsy and has an incidence among adults between $1: 500$ and $1: 1,000$ patientyears. ${ }^{8}$ The major risk factor for sudden unexpected death in epilepsy is chronic, uncontrolled epilepsy ${ }^{9,10}$. In addition, the major domain of potential mechanisms of sudden unexpected death in epilepsy is autonomic, i.e., cardiac abnormalities during and between seizures. ${ }^{9,11}$ Thus, although progress continues to be made in the field of

Tel.: 5511 5549-2064

Email: carlascorza.nexp@epm.br

Copyright (C) 2011 CLINICS - This is an Open Access article distributed under the terms of the Creative Commons Attribution Non-Commercial License (http:// creativecommons.org/licenses/by-nc/3.0/) which permits unrestricted noncommercial use, distribution, and reproduction in any medium, provided the original work is properly cited. sudden unexpected death in epilepsy in the last decade, the dearth of published data about the relationship between Down syndrome and sudden unexpected death in epilepsy encouraged us to address some questions regarding this specific topic.

As described previously, seizures in Down syndrome could be considered an epiphenomenon, i.e., they could be a consequence of the neurological alterations, both morphological and physiological, normally observed in this population. $^{12,13}$ Interestingly, the prevalence of epilepsy in individuals with Down syndrome increases with age, reaching almost $50 \%$ of all Down syndrome patients over 50 years of age. ${ }^{12}$ In general terms, approximately $8 \%$ of individuals with Down syndrome have seizure disorders that have been classified as partial seizures (47\%), infantile spasms $(32 \%)$ or generalized tonic-clonic seizures $(21 \%){ }^{12}$ Another interesting characteristic is the bimodal distribution of seizure onset: $40 \%$ of Down syndrome patients begin having seizures before the age of 1 year, and $40 \%$ experience seizures for the first time in the third decade of life. ${ }^{12}$ The occurrence of primarily infantile spasms predominates in the younger group, while partial seizures are more frequently observed in the older Down syndrome group. Tonic-clonic seizures seem to be equally distributed throughout life $\mathrm{e}^{12,14}$.

Taken together, these data raise the following question: Is there a risk of sudden unexpected death in epilepsy in individuals with Down syndrome who do not respond optimally to the treatment established by neurologists to control their seizures? Unfortunately, the answer is yes.

As previously mentioned, a number of associated factors for sudden unexpected death in epilepsy have been reported, but the results are not wholly consistent between studies. The factors examined include refractoriness of the epileptic condition, presence of generalized tonic-clonic seizures, early onset of epilepsy, antiepileptic medication (polytherapy with antiepileptic drugs), young age, and duration of the seizure disorder. ${ }^{9,10}$ In addition, although the different mechanisms may play separate roles in different cases, putative pathomechanisms include cardiac arrhythmia due to myocardial ischemia, electrolyte disturbances, arrhythmogenic drugs or transmission of epileptic activity via the autonomic nervous system to the heart. ${ }^{11}$ In evaluating the data of these conditions concurrently with data from Down syndrome patients with seizure disorders, we can assume that these individuals may be considered potential candidates for sudden unexpected death in epilepsy. Moreover, we cannot fail to mention that approximately $40 \%$ of individuals with 


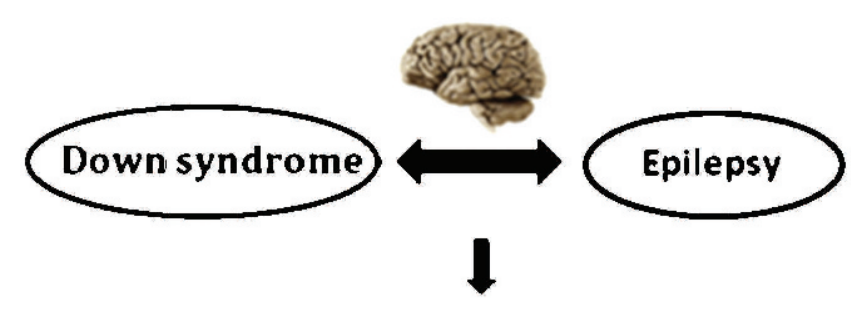

Uncontrolled seizures

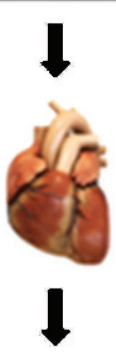

Cardiovascular abnormalities

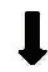

SUDEP

Figure 1 - The hypothesis that cardiovascular disturbances and hence sudden unexpected death in epilepsy, at least in some cases, could be related to the occurrence of refractory epilepsy in people with Down syndrome is proposed. Furthermore, assuming that $40 \%$ of individuals with Down syndrome have cardiovascular abnormalities, this fact would further facilitate the occurrence of a tragic eventin this specific population.

Down syndrome have congenital cardiac anomalies, ${ }^{15,16}$ doubling the chance of having a fatal event and redoubling our concern for these individuals. On the basis of this probability, how might we proceed

We are unable to prevent the occurrence of these illnesses or completely reverse the clinical picture when both have already occurred. However, a few simple, common, established strategies can be concurrently implemented by neuroscientists. First, it should be asked whether the search for some seizure-related event should be part of the clinical, routine checks in Down syndrome patients. Second, if this search is made, and considering that many individuals with Down syndrome have congenital heart defects, it should be asked whether palliative care of the cardiovascular system of these patients should be redoubled. To accomplish this redoubling, indispensable strategies, such as taking a detailed cardiovascular history and screening for symptoms of cardiovascular disease, cardiovascular risk factors and prior cardiac findings (electrocardiogram and echocardiogram), should be conducted frequently. Third, physicians who follow these patients should work collaboratively and should suggest that these patients be treated in a translational manner, i.e., that treatments should integrate advancements in basic science with clinical trials, taking research from "bench-to-bedside." 17 To do this effectively, some important well-established translational strategies ${ }^{18}$ should be adopted, such as maintaining a healthy weight, consuming a healthy and balanced diet, avoiding smoking, limiting alcohol consumption, maintaining regular physical activity (under the supervision of a qualified professional), maintaining good quality sleep and improving stress management by making time for relaxation and learning healthier coping strategies. Additionally, establishing a diet with omega-3 fatty acids from fish or fish oil supplements is strongly recommended.

On the whole, we strongly believe it is time to establish a taskforce to assess the state of knowledge regarding the occurrence of sudden unexpected death in epilepsy in Down syndrome, including clinical management, research directions, and educational, social and cultural efforts. To provide a better future for these patients, a list of the current problems should be compiled and analyzed to determine which feasibly-solved problems merit the most attention. Finally, neuroscientists must be faithful to concepts originating in eastern cultures: Great achievements are possible when you give importance to small beginnings.

\section{REFERENCES}

1. Pueschel SM. A historical view point. In: Pueschel SM, ed. Down syndrome growing and learning. (Human potentials for children series). Kansas City: Canning, Murphy, Zaumer, Andrews and McMeel, Inc. 1981. p. 37-9.

2. Korenberg JR., Pulst SM. Gerwehrs. Advances in the understanding of chromosome 21 and Down Syndrome. In: Lott I, McCoy E, editors. Down syndrome: Advances in medical care. New York: Wiley-Liss; 1992. p. 3-12.

3. Byard RW. Forensic issues in Down syndrome fatalities. J Forensic Leg Med. 2007;14:475-81, doi: 10.1016/j.jflm.2007.01.001.

4. Frid C, Drott P, Lundell B, Rasmussen F, Annerén G. Mortality in Down's syndrome in relation to congenital malformations. J Intellect Disabil Res 1999;43:234-41, doi: 10.1046/j.1365-2788.1999.00198.x.

5. Nei M, Hays R. Sudden unexpected death in epilepsy. Curr Neurol Neurosci Rep. 2010;10:319-26, doi: 10.1007/s11910-010-0116-4.

6. Epilepsy Atlas. Geneva, Switzerland: World Health Organization; 2005.

7. Duncan JS, Sander JW, Sisodiya SM, Walker MC. Adult epilepsy. Lancet. 2006;367:1087-100, doi: 10.1016/S0140-6736(06)68477-8.

8. Schuele SU, Widdess-Walsh P, Bermeo A, Lüders HO. Sudden unexplained death in epilepsy: the role of the heart. Cleve Clin J Med. 2007;74:S121-27, doi: 10.3949/ccjm.74.Suppl_1.S121.

9. Surges R, Thijs RD, Tan HL, Sander JW. Sudden unexpected death in epilepsy: risk factors and potential pathomechanisms. Nat Rev Neurol. 2009;5:492-504, doi: 10.1038/nrneurol.2009.118.

10. Tomson T, Nashef L, Ryvlin P. Sudden unexpected death in epilepsy: current knowledge and future directions. Lancet Neurol. 2008;7:1021-31, doi: 10.1016/S1474-4422(08)70202-3.

11. Stollberger C, Finsterer J. Cardiorespiratory findings in sudden unexplained/unexpected death in epilepsy (SUDEP). Epilepsy Res 2004;59:51-60, doi: 10.1016/j.eplepsyres.2004.03.008.

12. Menéndez M. Down syndrome, Alzheimer's disease and seizures. Brain Dev. 2005;27:246-52, doi: 10.1016/j.braindev.2004.07.008.

13. Romano C, Tine A, Fazio G, Rizzo R, Colognola RM, Sorge G et al., Seizures in patients with trisomy 21. Am J Med Genet Suppl 1990;7:298300.

14. Pueschel SM, Louis S, McKnight P. Seizure disorders in Down syndrome. Arch Neurol 1991;48:318-20.

15. Goodman R., Gorlin R. The Malformed Infant And Child: an Illustrated guide. New York: Oxford University; 1983. p. 122-3.

16. Barnett ML, Friedman D, Kastner T. The prevalence of mitral valve prolapse in patients with Down syndrome: implications for dental management. Oral Surg Oral Med Oral Pathol 1988;66:445-7, doi: 10. 1016/0030-4220(88)90264-2.

17. Goldblatt EM, Lee WH. From bench to bedside: the growing use of translational research in cancer medicine. Am J Transl Res. 2010;2:1-18.

18. Scorza FA, Arida RM, Terra VC, Cavalheiro EA. What can be done to reduce the risk of SUDEP? Epilepsy Behav. 2010;18:137-8, doi: 10.1016/ j.yebeh.2010.04.046. 